\title{
Führung und Sinn. Plädoyer für einen existenzanalytischen Umgang mit dem Sinnphänomen
}

\author{
Rüdiger Heinrich Jung ${ }^{1}$ \\ Online publiziert: 9. April 2020 \\ (c) Der/die Autor(en) 2020
}

\section{Zusammenfassung}

In diesem Beitrag der Zeitschrift „Gruppe. Interaktion. Organisation. (GIO)“ werden im Kontext der unmittelbaren Führungsbeziehung zwischen Vorgesetzten und Mitarbeitenden Sinn als Ergebnis werterealisierenden Verhaltens beschrieben, konkrete Werte- und Sinnpotenziale aufgezeigt und förderliche Rahmenbedingungen einer sinnorientierten Führung skizziert.

In der Diskussion um adäquate Formen von Führung und Organisation in der modernen Arbeitswelt erfährt das Sinnphänomen erhöhte Aufmerksamkeit. Dabei ist ein zum Teil vager, zum Teil großzügiger Umgang mit dem Sinnbegriff zu beobachten. Um Sinn als Motivationsfaktor sui generis nutzbar zu machen, plädiert der Beitrag für ein aus der anthropologischen Philosophie begründetes und dem existenzanalytischen Verständnis von Viktor E. Frankl entlehntes Sinnkonzept.

Schlüsselwörter Existenzanalyse · „Frankl“ · Sinn · sinnorientierte Führung · werteorientierte Führung · Werterealisierung

\section{Leadership and meaning. A plea for applying the framework of existential analysis to the phenomenon of meaning}

\begin{abstract}
In this article of the journal "Gruppe. Interaktion. Organisation. (GIO)" meaning is described in the context of direct relationships between supervisors and their employees as the result of value actualising behaviour; concrete potentials of value and meaning are demonstrated and conducive framework conditions for a meaning-oriented leadership are mapped out.

In the debate on adequate forms of leadership and organization in today's professional world, the phenomenon of meaning is receiving increased attention. In this context, a partly vague and partly generous handling of the notion of meaning can be observed. In order to utilise meaning as a motivational factor sui generis, the current contribution draws on an understanding of meaning based on anthropological philosophy and inspired by Viktor E. Frankl's concept of existential analysis.
\end{abstract}

Keywords Existential analysis · "Frankl" · Meaning · Meaning-oriented leadership · Value-oriented leadership · Value-actualisation

\section{Einleitung}

Führung und die damit verbundenen Willensbildungs- und Willensdurchsetzungsprozesse scheinen ihrer Funktion der Zielausrichtung und motivierten Zielverfolgung von und

Prof. Dr. Rüdiger Heinrich Jung

rhjung@rheinahrcampus.de

1 RheinAhrCampus, Hochschule Koblenz,

Joseph-Rovan-Allee 2, 53424 Remagen, Deutschland in Organisationen nicht mehr ausreichend gerecht $\mathrm{zu}$ werden. Die Komplexität, Mehrdeutigkeit und Änderungsdynamik von Handlungssituationen schwächen die Überzeugungskraft von Führungskommunikation und erzeugen Unsicherheit bei allen Beteiligten. Nicht selten bereitet der 
Transparenzverlust bei komplexen Zusammenhängen zudem das Feld für Führungswillkür und die Instrumentalisierung von Führung für egoistische Motive der Führungspersonen. Am augenfälligsten ist das derzeit auf der gesellschaftlich-politischen Ebene. Aber auch auf der uns hier interessierenden betrieblichen (organisationalen) Ebene nagen schwierige Zeiten am Vertrauen gegenüber gewohnten Mustern und rufen nach neuen Lösungen. Manche Lösungsvorschläge sind bloße Wortakrobatik - statt ,Führung ' wird ,Leadership“ gefordert, statt Führungspersonen ,richtige“ ,Leader ${ }^{.}{ }^{1}$ Anderes scheint beachtenswert. Im Spektrum des Beachtenswerten markieren die Forderung nach Führungsersatz durch Selbststeuerung (,unboss the company“, wie Novartis-Chef Vasant Narasimhan es nennt) und die Empfehlung einer sinn-vollen Aufladung der Führungsfunktion, ein „Führen mit Sinn“, besondere Positionen. Die Aufladung von Führung in Organisationen durch das Sinnphänomen soll der Betrachtungsgegenstand des vorliegenden Beitrags sein. Nach einer Klärung des zugrundeliegenden Sinnverständnisses geht es vor allem um ein Aufzeigen der Sinnpotenziale in der unmittelbaren Führungsbeziehung. Damit sprechen wir jede Führungskraft mit ihren Möglichkeiten einer sinnorientierten Führung an.

\section{2 "Sinn" und seine motivationstheoretische Einordnung}

\subsection{Sinn als individuelles und situatives Wertfühlen der geistigen Person}

„Sinn“ als tief in der deutschen Sprache verwurzelter Begriff für den „Weg hin zu etwas“ wurde bereits früh von der materiell-räumlichen in die geistige Sphäre für die „Beziehung auf etwas“ übertragen (Grimm und Grimm 1999 [1984]). Maurice Merleau-Ponty verknüpft dieses Sinnverständnis mit dem Merkmal der Transzendenz: „Allen Bedeutungen des Wortes ,Sinn ' zugrunde liegend finden wir den einen Grundbegriff eines Seins, das auf etwas hin, was es nicht selber ist, orientiert oder polarisiert ist, und alles verweist uns so auf den Gedanken des Subjekts als Ek-stase (aus sich heraustreten, außer sich sein; Anm. d. Verf.) und auf ein aktives Transzendenzverhältnis zwischen Subjekt und Welt" (Merleau-Ponty 1966, S. 488f.). Für Viktor E. Frankl, der mit seinen Arbeiten die „Logotherapie“ (griechisch „logos" mit einer weiten Bedeutung, darunter „Geist“, „Sinn“) als sinnorientierte Psychothe-

\footnotetext{
1 „Das Ausweichen auf die neudeutsche Vokabel „Leadership“ illustriert, wie die semantische Problematik übertüncht und die Herausbildung eines angemessenen Führungsverständnisses erschwert wird.“ (Bundesverband der Personalmanager: Führung in der Krise. 10 Thesen; https://www.bpm.de/thesen_fuehrung_in_der_krise, abgerufen am 17.09.2019).
}

rapie, als „Psychotherapie vom Geistigen her“ (Frankl 2007, S. 45), begründet hat, scheint in diesem Transzendenzverhältnis das eigentlich Menschliche auf: SelbstTranszendenz menschlicher Existenz ist der „Tatbestand, daß Menschsein immer über sich selbst hinaus auf etwas verweist, das nicht wieder es selbst ist, - auf etwas oder auf jemanden: auf einen Sinn, den da ein Mensch erfüllt, oder auf mitmenschliches Sein, dem er da begegnet.“ (Frankl 2007, S. 213).

Frankl orientiert sich bei seiner existenzanalytisch, d.h. für ihn auf geistige Existenz hin begründeten Lehre (siehe Frankl 2010, S. $57 \mathrm{ff}$.) vor allem an der philosophischen Anthropologie von Max Scheler mit ihren Überlegungen zum geistigen Sein des Menschen und dessen Wertebezogenheit. Der geistigen Dimension von Menschsein ist die Sinnfrage inhärent. Geistiges Sein ist für Scheler klar zu trennen von der körperlichen (physiologischen) und seelischen (psychologischen) Zuständlichkeit des menschlichen Organismus. Das Geistige ist die weltoffene und weltbezogene Wesenstatsache von Menschsein (vgl. Scheler 2010, S. 28). Dieser Weltbezug der geistigen Person ist für Scheler eine Wertebezogenheit, ja Wertestrebigkeit (zum Streben nach Werten siehe Schelers Hauptwerk 1927: passim, insbes. $28 \mathrm{ff}$.). Ähnlich konstatiert Nicolai Hartmann: „Der Mensch ist das den Werten als solchen grundsätzlich zugewandte, den Unwerten grundsätzlich abgewandte Wesen." (Hartmann 1962, S. 159) Werte als Objekte idealen Seins sind zunächst einmal bloße Möglichkeiten, die durch Akte der Werterealisierung - beispielsweise durch Akte der Liebe, der solidarischen Unterstützung, des Tätigseins für eine wertvolle Idee oder Sache oder durch eine würdevolle Haltung - erlebbar (,Wertfühlen“, Scheler 1927, S. 30 ff., 264 ff., 358) werden. Und eben jene Vorgänge der Werterealisierung sind für Frankl die situativen Ereignisse, die mit einer Sinnwahrnehmung, einem Fühlen von Sinn, einhergehen. ,Sinn ist also jeweils der konkrete Sinn einer konkreten Situation.“ (Frankl 2006, S. 157) Sinn ist „die wertvollste Möglichkeit in einer Situation“, so Längle (2008, S. 56) mit einer gewissen Steigerung des Anspruchs an die Werterealisierung.

Wert- und Sinnfühlen setzten im Übrigen nicht, wie häufig unterstellt, eine Ziel- oder Zwecksetzung voraus. Voraussetzung ist das Sich-anfragen-lassen von einer gegenwärtigen Situation und der mit ihr verbundenen Wertepotenzialität. Kein Einkaufsbummler, der einem gestürzten Passanten zur Hilfe eilt, hat seinen Einkaufsbummel mit der Zielsetzung einer Erste-Hilfe-Leistung angetreten. In der Konsequenz des hier vorgetragenen Sinnverständnisses müssen die weiteren Ausführungen stets das Hin-zu-Werten als empirische Manifestation geistigen Seins beachten (zur Unterscheidung von dem Sinnverständnis in den Sozialwissenschaften, das jedweder sozialen Beziehung Sinn zuschreibt, siehe Jung 2020). 


\subsection{Motivationstheoretische Einordnung von Sinn}

Motivationstheoretisch ist die Aktivierung des „Willens zum Sinn“ (Frankl 2007: passim, z.B. S. 111) über eine situativ gebotene Möglichkeit zur Werterealisierung erklärbar. Voraussetzung ist die „Wertsichtigkeit“ (Hartmann 1949, S. 116) der geistigen Person, d.h. die Fähigkeit, den Wertegehalt einer Situation zu erspüren. In der Philosophie wird die Wertsichtigkeit als intuitiver, analytischer Erklärung wenig zugänglicher Vorgang gesehen, „eine Erfahrungsart, deren Gegenstände dem ,Verstande“ völlig verschlossen sind; für die dieser so blind ist wie Ohr und Hören für die Farbe“ (Scheler 1927, S. 262). Kuhl verweist in seinem Grundlagenwerk zur Persönlichkeitspsychologie bei der Einordnung von Frankls logotherapeutischem Ansatz auf eine ,ganzheitlich-intuitive Intelligenz“ (Kuhl 2010, S. 325) und deren Potenzial zum Sinnerleben.

Seine Entbundenheit vom psycho-physischen Zustand des Menschen gibt dem Willen zum Sinn auch unter dem Aspekt der Motivation eine Sonderstellung, die sich in Extremsituationen menschlicher Existenz besonders eindrucksvoll beobachten lässt (siehe Jung 2019, S. 74 ff.). Die Sinnstrebigkeit des Menschen existiert unabhängig vom Stand der Befriedigung sonstiger Bedürfnisse, kann deshalb als Motivationsfaktor sui generis betrachtet und auch nicht mit der Vorstellung einer hierarchischen Ordnung wie etwa in der Motivationstheorie Abraham H. Maslows (,hierarchy of needs“; Maslow 1954, S. 97 ff.) verknüpft werden. In einer Würdigung der bekanntesten Inhalts- und Prozesstheorien der Motivation sieht Zaiser grundsätzliche Verknüpfungsmöglichkeiten mit dem Willen zum Sinn, muss aber zugleich konstatieren, dass dieser doch - mit der Ausnahme von Maslow, der Frankl in der Bedeutung des Willens zum Sinn ausdrücklich zustimmt (siehe Maslow 1966, S. 108) - „stets unreflektiert blieb.“ (Zaiser 2004, S. 76) „Sie nehmen einzig den psychischen Unterbau wahr, nicht aber die existentiale Ausgerichtetheit des Menschen auf Sinn" (Zaiser 2004, S. 78), d.h. das Geistige in der menschlichen Handlungsbegründung bleibt außen vor. Jung et al. erkennen zumindest in dem, auf den Arbeitskontext bezogenen, „Job Characteristics Model“ von Hackman/ Oldham eine Nähe zu Frankls Sinnverständnis, weil über die Merkmale Vielfalt, Ganzheitlichkeit und Bedeutung einer Tätigkeit die empfundene Sinnhaftigkeit der Arbeit und darüber die intrinsische Arbeitsmotivation beeinflusst wird. „Die vielfältige, ganzheitliche, bedeutungstransparente Tätigkeit enthält für jeden Mitarbeiter ein hohes Potenzial, sich im Tätigsein für etwas Wertvolles außerhalb seiner selbst zu finden.“ (Jung et al. 2018, S. 218).

Den Zusammenhang von Wertestrebigkeit und Motivation hat bereits Scheler in seinen anthropologischen Arbeiten dargelegt. Er spricht von „Wertfühlen (Motivationsgefühl), als der Quelle des Strebens,“" (Scheler 1927, S. 358) und verdeutlicht den motivatorischen Gehalt des Hingezogenseins des Menschen zu Werten mit dem Begriff der „Zugkausalität" (Scheler 1927, S. 357). Entsprechend bescheibt Frankl in seiner existenzanalytischen Betrachtung Sinn und Werte als „das, was den Menschen von außen anzieht“" (Frankl 2006, S. 58) und kritisiert dabei die diesbezügliche motivationstheoretische Blindheit der klassischen Psychologie.

Bezogen auf das Leben allgemein und den Arbeitskontext speziell mehren sich in den letzten Jahren wissenschaftliche Beiträge, die Sinn in den Mittelpunkt ihrer Betrachtung stellen. Wenn dabei das motivatorische Potenzial von Sinn häufig ohne weitere Begründung unterstellt oder vorausgesetzt wird, darf die Schelersche ,Zugkausalität" stets mitgedacht werden. Ausdrücklich enge Bezüge zum Werk Frankls und dem Motivationsverständnis von Sinn finden sich beispielsweise in den Arbeiten von Graf (2007), Fintz (2014) und Pircher-Friedrich (2019). Schnell (2016, S. 164) sieht aufgrund eigener empirischer Studien Sinnerfüllung und Arbeitsengagement als die ,zwei Seiten einer Medaille“: „Während Arbeitsengagement die affektive Seite darstellt, also Gefühle der Lebendigkeit und Begeisterung, repräsentiert berufliche Sinnerfüllung die kognitiv-evaluative Seite“.

\section{Sinnorientierung in der Führungsbeziehung}

\subsection{Zum Umgang mit dem Sinnphänomen im Kontext von Arbeit, Management und Führung}

Unter den frühen Plädoyers im deutschsprachigen Raum, Motivation im Lichte der existenzanalytischen Begründungen Viktor E. Frankls auch im Kontext von Arbeit, Organisation und Führung neu zu denken, ist dasjenige von Fredmund Malik ein besonders markantes. Malik hält „die Theorie Frankls für das Beste, was je über Motivation gesagt wurde." (Malik 1997, S. 38; siehe auch Tschirky 1991) Für die lange Zeit in der Breite ausgebliebene Resonanz gibt es verschiedene Gründe: Die Grundlagen des Sinnverständnisses von Frankl sind in der Arbeits- und Organisationspsychologie alles andere als „mainstream“. Das mag unter anderem daran liegen, dass Sinn als geistiges Phänomen in dem Transzendenzverhältnis von Subjekt und Welt schwer fassbar und für jeden Operationalisierungsversuch problematisch ist. Hinzu kommt, dass Frankl in der Konsequenz seines Verständnisses von Sinnerleben so sehr auf die Freiheit und Verantwortung des (Sinn suchenden und findenden) Individuums pocht, dass dies im vorherrschenden Fremdsteuerungsparadigma von Arbeit, Organisation und Führung eher befremdlich wirkt. In irreführenden Formulierungen wie „Sinn stiften“ und „Sinn geben“ scheint 
dieses Paradigma - selbst in ausdrücklichen Bezügen auf Frankls Werk - bis heute auf.

Im Zuge der Diskussion über Veränderungen in der Arbeitswelt und deren Auswirkungen auf Führung erfährt das Sinnphänomen eine deutlich gestiegene Aufmerksamkeit, was aber nicht gleichzusetzen ist mit einer Rezeption des hier zugrunde gelegten Sinnverständnisses. Der Deutungsraum des Phänomens und die angesprochene schwierige Fassbarkeit ermöglichen einen großzügigen Umgang mit dem Sinnbegriff. Manchmal wird dabei unter Berufung auf die Sinnkonzeption Frankls ein weiter Bogen gespannt bis hin zu ,meaning in society“ und einem ,,meaningful capitalism" (Pattakos und Dundon 2017, S. 46, 48). Für den hier interessierenden Kontext sind eher andere Beobachtungen relevant.

$\mathrm{Da}$ ist zum einen die Tendenz, unter weitgehender Ausblendung wissenschaftlicher Begriffsbildung, Zusammenhangsbeschreibung und Zusammenhangserklärung den Sinnbegriff für eine normative Deklamation von Werten für die zukunftsfähige Organisation zu nutzen. Die Arbeit von Anja Förster \& Peter Kreuz mit ihren „sechs Sorten Sinn“ - Perfektion, Innovation, Herausforder-Rolle annehmen, Demokratisierung, Verantwortung, menschliche Werte (Förster und Kreuz 2009, S. 29 ff.) sei hier beispielhaft erwähnt.

$\mathrm{Da}$ ist zum anderen der Versuch, über einen narrativen Ansatz der Darlegung interessanter praktischer Beispiele ein neues Denken für Organisation und Führung zu befördern, wobei „sinnstiftenden Formen der Zusammenarbeit" (Laloux 2015) eine besondere Bedeutung zukommt. Die durchaus anregende Arbeit von Frederic Laloux sei beispielhaft genannt für dieses narrative Vorgehen. Ihre Idee „eines evolutionären Sinnes“ (Laloux 2015, S. 193 ff.) bleibt vage, wobei die Hinweise auf ein „Hören“ und „Spüren“ (ebenda: $200 \mathrm{ff}$.) einen Bezug zur geistigen Dimension vermuten lassen.

$\mathrm{Da}$ ist zum weiteren ein großzügiger Umgang mit dem Sinnbegriff durch eine Anbindung oder gar Gleichsetzung mit anderen, wohl eingeführten Begriffen. Auffällig ist eine häufige Anbindung an die Begriffe „Zweck“ und ,Ziel“. Dabei werden „Sinn, Purpose und Zweck ... weitgehend als Synonyme“ (Fink und Moeller 2018, S. 24) verwendet oder Sinn wird als ,,auf übergeordnete Ziele“ (Schnell 2016, S. 27) ausgerichtet beschrieben. ,So wird aus sinnloser Bewegung sinnvolle Bewegung, wenn sie Zielen wie Gesundheit oder Fitness dient." (Schnell 2016, S. 27). Das ermöglicht zwar die Übertragung des Sinnbegriffs auf kollektive Gebilde (z.B. Organisationszweck, -ziel, -sinn), verkennt aber das unstrittige allgemeine Verständnis, dass Ziele und Zwecke gesetzt, für verbindlich erklärt, delegiert sowie auf logische Mittel-Zweck- bzw. Ober- und Unterzielbeziehungen hin analysiert werden können. Das ist mit dem weiter oben dargelegten Verständnis von Sinn schlecht vereinbar (zur Unterscheidung von Zwecken und Werten siehe tiefer gehend Scheler 1927, S. 25 ff.; mit Bezug auf Frankls Sinnverständnis z. B. Berschneider 2003, S. 42f.).

Fruchtbar für die weitere Erkenntnisentwicklung dürften Arbeiten zur empirischen Begründung von Sinn(erleben) im Arbeitskontext sein. Abgesehen von der grundsätzlichen Problematik einer empirischen Begründung geistiger Phänomene wie Wertfühlen und Sinn unterscheiden die eingesetzten Erhebungsinstrumente aber häufig nicht zwischen Sinnerleben am Arbeitsplatz und Sinn im Leben allgemein; zudem heben sie stellenweise auf Wahrnehmungen ab, die eher psycho-soziales Wohlbefinden statt geistiges Sinnfühlen repräsentieren. Dennoch sei nachfolgend auf einige interessante Ergebnisse verwiesen.

Tatjana Schnell konstatiert positive Korrelationen zwischen Sinnerfüllung am Arbeitsplatz und Arbeitszufriedenheit, Leistung, organisationaler Bindung sowie negative Korrelationen bezüglich Fluktuation, innerer Kündigung, Zynismus, Erschöpfung und Stresserleben (Schnell 2016, S. 164 mit Verweis auf eine Mehrzahl europäischer und nordamerikanischer Studien). Ähnlich konstatiert Bernhard Badura für die „erlebte Sinnhaftigkeit“ am Arbeitsplatz „erhebliche Auswirkungen auf das Wohlbefinden und auf Qualität und Umfang der erbrachten Leistung" (Badura 2018, S. 1) sowie auf Gesundheit insgesamt (ebenda: S. 5), wobei er die Erforschung dieser Zusammenhänge „,noch in den Anfängen“ (ebenda: S. 2) sieht.

Mit Bezug auf die Frage, wie Sinnerleben im Arbeitskontext entsteht, identifiziert Schnell aufgrund eigener empirischer Untersuchungen vier „Prädiktoren beruflicher Sinnerfüllung" (Schnell 2016, S. $156 \mathrm{ff}$.; Schnell et al. 2013). Der Prädiktor „Bedeutsamkeit der eigenen Tätigkeit“, definiert als das Ausmaß des wahrgenommenen Nutzens der eigenen Tätigkeit für andere Menschen, weist dabei den deutlichsten Bezug zur Variable ,sinnvolle Arbeit“ (,,meaning in work") auf. Wir sehen in diesem „Prädiktor" ein eigentliches Maß für das Sinnempfinden bei der Arbeit, weil er ganz unmittelbar für die wertestrebige Hinwendung zu etwas, was nicht ich (die befragte Person) selbst bin, steht. Mit den übrigen Prädiktoren „selbsttranszendente Unternehmensorientierung “, ,sozio-moralische Atmosphäre“ und ,Job-Passung“ sind mögliche Einflussfaktoren abgebildet, die nicht zwingende Voraussetzungen des individuellen und situativen Sinnempfindens sind, aber dieses doch begünstigen können. Ronald Busse subsummiert in seiner methodisch interessanten Modellierung von Sinnzusammenhängen psychologische (z. B. Zufriedenheit, Wertschätzung durch Kollegen) wie noologische (z.B. Bedeutung der eigenen Arbeit für die Umwelt, ethische Vorbildlichkeit) Elemente unter „Hauptkategorien des Willens zum Sinn“ (Busse 2019, S. 133). In der zugrundeliegenden empirischen Studie erwies sich die Vorgesetzten-Mitarbeiter-Beziehung als bedeutsamster Einflussfaktor für das Sinnemp- 
finden - übereinstimmend für in Deutschland, den USA, China, Indien und Südkorea erhobene Daten (Busse et al. 2018, S. 67 f.).

Eine fehlende Striktheit in dem Auseinanderhalten von psychologischer und noologischer Dimension eignet vielen Untersuchungen zu unserem Thema, auch den zuvor genannten empirischen Studien. Da der Mensch aus beiden Seins-Dimensionen heraus und nicht selten auch ohne ein Bewusstsein für diese Unterscheidung handelt, mag man das für theoretisch unsauber, aber praktisch unerheblich halten. Einem guten Verständnis der Besonderheit des Sinnphänomens, für das Führungskräfte sensibilisiert werden sollen, dient der großzügige Umgang mit dem Sinnbegriff indes nicht.

Für die weitere Betrachtung von Führung und Sinn in Organisationen soll nun konkretisiert werden: Wir beziehen uns auf die durch unmittelbare Führungsbeziehungen gekennzeichneten organisationalen Subsysteme (Teams, Gruppen, Abteilungen, „Führungssegmente“ - zum Begriff siehe Seidel 1978, S. 116f.) und knüpfen damit an die erkannte Bedeutung der Führungsbeziehung (siehe die oben erwähnte Arbeit von Busse et al. 2018; siehe auch den Beitrag von Felfe et al. 2018) für das Sinnerleben im organisationalen Kontext an. Für das einzelne Organisationsmitglied als „Träger“ subjektiver Geistigkeit und damit auch Sinnbedürftigkeit finden auf dieser Handlungsebene die täglichen Konkretisierungen von Sinn statt (oder auch nicht). Diesen Konkretisierungen als Möglichkeiten der Werterealisierung im Einflussbereich jeder Führungskraft gilt unser hauptsächliches Augenmerk. Damit soll die Übertragung des Themas „Führung und Sinn“ auf die gesamtorganisationale Ebene in ihrer möglichen Bedeutung ebenso wenig bestritten werden wie die rahmengebende Bedeutung der Makroebene für das Geschehen auf der Mikroebene. Mit Blick auf die angesprochenen großzügigen Interpretationen von Sinn bietet die hier gewählte Fokussierung aber genügend Klärungspotenzial.

\subsection{Sinn im Kontext der unmittelbaren Führungsbeziehung}

Wir haben Sinn als individuelles und situatives Wertfühlen der geistigen Person definiert. Der Mensch fühlt dann den Sinn seines Seins, wenn per Haltung und Tat die Wertepotenzialität einer Situation in eine Werteverwirklichung transformiert wird, was für ihn und möglicherweise andere an der Situation Beteiligte zu einem Wertfühlen führt. Bei einer Würdigung der Sinnorientierung im Kontext der unmittelbaren Führungsbeziehung sind zwei relevante Sichtweisen zu unterscheiden:

1. die Sicht auf die Werteverwirklichung und das damit verbundene Sinnerleben der Mitarbeiterinnen und Mitarbei- ter und die Frage, in welcher Weise die zuständige Führungskraft diese beeinflussen kann

2. die Sicht auf die Werteverwirklichung und das damit verbundene Sinnerleben der jeweiligen Führungskraft und die Frage, inwieweit für die Führungsperson selbst die Führungsarbeit ein Potenzial für Sinnerleben beinhaltet.

In der folgenden Betrachtung bedienen wir uns der von Viktor E. Frankl vorgenommenen Unterscheidung in die Kategorien schöpferische Werte, Erlebniswerte und Einstellungswerte - von ihm bezeichnet als ,drei Hauptstraßen ..., auf denen sich Sinn finden lässt“ (Frankl 2006, S. 47).

\subsubsection{Möglichkeiten der Werterealisierung auf der Mitarbeiterebene}

Schöpferische Werte können durch das Hervorbringen einer materiellen oder immateriellen Leistung verwirklicht werden: ein Mitarbeiter der Betriebstechnik, der den Kolleginnen und Kollegen in der Produktion eine zuverlässige und sichere Maschine hinstellt; eine Pflegerin, die einem Patienten einen Spaziergang im Park ermöglicht; der Umweltaktivist, der ein Protesttransparent an einem Trawler befestigt. Die Werteverwirklichung hängt nicht - wie manchmal unterstellt wird - zwingend davon ab, dass die jeweils tätige Person dabei ihre besonderen Fähigkeiten und Begabungen einbringen kann. Werteberührung und wertebezogenes Handeln können trotz Unsicherheit, geringer Befähigung, vielleicht sogar Versagensangst geschehen. Soweit auf den Zweck einer Organisation und das damit einhergehende Sinnpotenzial abgehoben wird, ist - hier am Beispiel eines Pharmaunternehmens - anzumerken: Für die Werteberührung und das mit ihr einhergehende Motivations- und Sinnpotenzial ist es erforderlich, dass die Mitarbeiterin oder der Mitarbeiter einen Zusammenhang zwischen der eigenen Tätigkeit und der Verfügbarkeit des Medikaments für Erkrankte wahrnimmt. Laloux spricht davon, dass ,der individuelle Sinn und der Sinn der Organisation miteinander in Resonanz treten“ (Laloux 2015, S. 221; zur Bedeutung der „Passung von organisationalem und Individual-Sinn“ siehe auch Brohm 2017, S. 37, 41 ff.).

Erlebniswerte werden über ein aufnehmendes, das Werteempfinden berührendes Erleben des Arbeitsgeschehens verwirklicht. Hier geht es nicht um das eigene schöpferische Tätigsein, sondern um ein hinwendendes Beteiligtsein: an der Art und Weise des Miteinanders am Arbeitsplatz, der wahrgenommenen Gerechtigkeit bei der Verteilung von Arbeitslast, der gegenseitigen Rücksichtnahme und Unterstützung, Offenheit und Ehrlichkeit, der wahrgenommenen Bedeutung von Arbeitsqualität, des wertschätzenden Umgangs mit Ressourcen u.a.m. Bei den Erlebniswerten wird besonders deutlich, dass die Wahrscheinlichkeit der Berührung von einem Wert beeinflusst wird vom individuellen Werte- 
system eines Menschen. Wer Gerechtigkeit oder gegenseitige Unterstützung oder Arbeitsqualität nicht für wertvoll hält, wird davon möglicherweise auch nicht berührt werden - ausgeschlossen ist das indes nicht, sonst wären Veränderungen im individuellen Wertesystem gar nicht möglich.

Einstellungswerte werden durch persönliche, möglicherweise einer körperlichen oder psychischen Beschwernis trotzende, Haltungen verwirklicht: die Übernahme der Verantwortung für einen Fehler trotz der Möglichkeit, sich hinter der Mehrdeutigkeit der Fehlerzurechnung zu verstecken; das Vorleben von Teamgeist trotz wahrgenommener Egoismen bei allen anderen Beteiligten; die Aufnahme einer Arbeitstätigkeit trotz einer nur geringen finanziellen Besserstellung gegenüber der sozialen Absicherung in der Arbeitslosigkeit u.a.m. Einer bedrückenden Situation ein „Trotzdem“ entgegenzustellen, ist selbst bei fehlender Möglichkeit zur Realisierung schöpferischer Werte und Erlebniswerte eine der geistigen Person innewohnende Sinnmöglichkeit.

Die exemplarische Beschreibung von Werteverwirklichungen und damit verbundenem Sinnempfinden in der Mitarbeiterrolle lässt erkennen, dass Führung hierfür keine zwingende Voraussetzung ist. Andererseits kann nicht unterstellt werden, dass in der Konstellation ,,Vorgesetzte und Mitarbeitende" Werterealisierung und Sinnfühlen erschwert werden. Es hängt vom Verhalten der jeweiligen Führungsperson ab. Die Führungsrolle mit ihren Sinnmöglichkeiten soll deshalb im nächsten Abschnitt betrachtet werden. Auf eine erneute exemplarische Abhandlung der verschiedenen Wertekategorien wird dabei verzichtet.

\subsubsection{Möglichkeiten der Werterealisierung in der Führungsrolle}

Jede Führungsrolle ist konstitutiv durch ein Hin-zum-Anderen definiert, was den Werte- und Sinnaspekt geradezu einzuschließen scheint. Aber auch Führende haben wie jeder Mensch die Eigenschaft, in ihrem Handeln nicht nur ein Hin zu etwas, was nicht sie selbst sind, sondern auch eine Selbstbezogenheit gelten zu lassen. Die Erfahrung des Verfassers aus der Arbeit als Coach lehrt die Bedeutung der Frage: „Wozu ist es gut, dass gerade Sie (die vor mir sitzende Führungskraft) die Führungsaufgabe übernehmen sollten oder übernommen haben?" Wenn in den Antworten auf diese Frage nicht die Mitarbeitenden vorkommen, sondern das Ego die Antworten dominiert (z.B. „,weil ich weiterkommen möchte“, ,weil ich als Dienstältester einfach dran war"), ist offenkundig, dass für sinnorientierte Führungsarbeit ein erheblicher Coaching-Aufwand ansteht.

Mit Bezug auf Mitarbeiterinnen und Mitarbeiter lässt sich das Potenzial für Werterealisierung und Sinnfühlen in der Führungsrolle in zwei Arten unterteilen (von einer Weitung des Fokus auf Handlungsbezüge einer Führungskraft in Richtung anderer Personen innerhalb und außerhalb einer Organisation sehen wir hier ab):

\section{a. Beiträge der Führungsperson zu den Werterealisierun- gen und Sinnempfindungen der Mitarbeitenden}

Beiträge ergeben sich unmittelbar aus den zuvor (Abschn. 3.2.1) für die Mitarbeitenden beispielhaft beschriebenen Möglichkeiten der Werterealisierung. Wenn Führung einen Beitrag dazu leistet, dass Mitarbeitende den Wert ihrer Haltungen oder ihres Tätigseins wahrnehmen können, wenn Führung wertebezogenes Handeln unterstützt oder ermöglicht, kann sich auch auf Seiten der Führungsperson Sinnfühlen einstellen. „Gut, dass ich durch mein Führungshandeln Anderen (hier: Mitarbeitende, Teammitglieder u.ä.) einen Zugang zum Wertfühlen ermöglicht habe." So oder so ähnlich ließe sich - mit aller Unzulänglichkeit der Verbalisierung subjektiver Empfindungen - dieses Sinnfühlen bei der Führungsperson beschreiben. Den späteren Überlegungen (Abschn. 3.2.3) vorausgeschickt sei hier: Dieses Ermöglichen funktioniert nicht über einen Appell, eine Anweisung oder gar ein Einimpfen von Werten und deren Sinnpotenzial (,Das muss doch für Dich Sinn machen!“‘).

\section{b. Beiträge der Führungsperson zu einem guten Zustand des Psychophysikums der Mitarbeitenden}

Da hier das Sinnempfinden der Führungsperson und nicht das der Mitarbeitenden betrachtet wird, ergibt sich eine weitere Art von Sinnpotenzialität: Ein Wert- und Sinnfühlen kann sich bei der Führungsperson auch dadurch einstellen, dass über die Führungsarbeit ein Beitrag zu einem guten Zustand des Psychophysikums der Mitarbeitenden geleistet wird - beispielsweise durch eine ergonomische Arbeitsplatzgestaltung, den Einsatz für eine leistungsgerechte Entgeltanpassung oder die Berücksichtigung persönlicher Entwicklungsinteressen der Mitarbeitenden. Auch dies sind Führungsakte aus der Verantwortlichkeit für jemand Anderen, Handlungen aus dem personalen Geist und seiner Wertestrebigkeit. „Gut, dass ich durch mein Führungshandeln für Andere (hier: Mitarbeitende, Teammitglieder u.ä.) einen Beitrag $\mathrm{zu}$ einem gesunden, sicheren, angstfreien usw. Arbeitsplatz leisten kann." In diesem Empfinden liegt Sinn- und Motivationspotenzial für die Führungsperson selbst, weil der Wert des eigenen Tätigseins als Führungsperson erlebt wird. „Wann ist Führung sinnvoll?“, hat Hugo Tschirky bereits 1991 gefragt, und auch geantwortet: „... wenn sie die eigene Sinnfindung und gleichzeitig eine Sinnvermittlung an andere ermöglicht." (Tschirky 1991, S. 28).

In einem weiten Verständnis kann ,sinnorientierte Führung“ die zuvor unter (a) und (b) beschriebenen Aktivitäten der Führungsperson meinen. In einem engen Verständnis meint ,sinnorientierte Führung“ nur die unter (a) be- 
schriebenen Aktiväten, weil nur diese unmittelbar darauf abzielen, für die geführte(n) Person(en) die Berührung mit Werten, ein Wert- und Sinnfühlen im Zuge ihres Tätigseins $z u$ unterstützen oder zu ermöglichen. Diesem engeren Verständnis folgen die weiteren Überlegungen.

Bevor wir im nächsten Kapitel auf die grundsätzlichen Möglichkeiten sinnorientierter Führung eingehen, sei an einem kleinen Beispiel verdeutlicht, wie sich eine Führungsperson in ihrem Führungshandeln von einer situativen Sinnanfrage leiten lässt:

Gedacht sei eine Organisationseinheit mit einer Vorgesetzten und vier Mitarbeitern, beispielsweise ein Forschungs- und Entwicklungslabor mit der Laborleiterin und vier Laboranten. Im Zusammenhang mit der Vorbereitung eines Kundenbesuchs im Labor sind unter den Mitarbeitern wenig beliebte Aufräum- und Säuberungsarbeiten durchzuführen. Die Vorgesetzte beobachtet, dass einer der vier Laboranten sich nicht an der Aufräum- und Säuberungsarbeit beteiligt, sondern an seinen üblichen Materialversuchen arbeitet. Die drei Kollegen kommen auch so zurecht; möglicherweise ist es ihnen sogar ganz recht, dass ihr ohnehin als Querulant bekannter Kollege nicht mitmacht. Die Vorgesetzte könnte es laufen lassen und sich ein besonderes Dankeschön an die drei „Aufräumer" vornehmen. Schließlich weiß sie, dass die Führungskommunikation mit dem Querulanten oft stressig wird. Außerdem arbeitet dieser an einer Testreihe, deren Ergebnisse die Vorgesetzte sehr interessieren. Dennoch geht sie auf den Mitarbeiter zu und weist diesen an, sich umgehend an den Aufräumarbeiten zu beteiligen, weil es ihr in der so entstandene Situation zuoberst um den Wert der „Gerechtigkeit“ geht, für dessen Beachtung sie sich in ihrer Vorgesetztenfunktion zuständig und verantwortlich sieht. Obwohl es eine Möglichkeit gäbe, unter Verweis auf die Bedeutung der Testarbeiten dem möglicherweise unangenehmen Führungsgespräch aus dem Weg zu gehen, entscheidet sie sich für diese Werterealisierung, gestützt auf ihre Einflussmacht als Vorgesetzte. Mit ausdrücklichem Verweis auf die „Gerechtigkeit“ begründet sie auch ihre Anweisung.

Für die Vorgesetzte ist dies wertebezogenes, sinnorientiertes Führungshandeln. Es ist ein Beispiel für die Bedeutung von Führung, ,soziale Situationen, in denen sie (die Führungspersonen, Anm. d. Verf.) selbst mit enthalten sind, kontextbezogen zu steuern" (Geramanis 2016, S. 47), wobei die Fähigkeit der Wertsichtigkeit (siehe oben Abschn. 2.2) eine entscheidende Rolle spielt. Soweit die an dieser Situation beteiligten Personen einen Zugang zum Wert „Gerechtigkeit“ haben, stellt sich ein diesbezügliches Wert- und Sinnfühlen ein - bei der Führungsperson, die durch ihr Han- deln einem Wert zu Geltung verholfen hat (Werteverwirklichung), bei dem „Drückeberger“, der (vielleicht) erkennt, dass es etwas Wertvolleres als die Vermeidung der unangenehmen Arbeit gibt, und bei den übrigen Mitarbeitenden, für die „Gerechtigkeit“ zu einem Erlebniswert am Arbeitsplatz geworden ist. Sinnfühlen ist ein subjektiver Vorgang; insoweit kann hier nur die Möglichkeit und nicht eine Gewissheit formuliert werden.

Soweit unsere Darlegung einer sinnorientierten Führung, welche die je konkreten Wertemöglichkeiten einer Situation erkennt und nutzt. Konsequenterweise ist dieses situative Handeln eingebettet in eine grundsätzliche, der Werte- und Sinnorientierung Raum gebende Gestaltung der Führungsbeziehung - eine sinnorientierte Arbeits- und Führungskultur. Einige Ansatzpunkte hierfür sollen nachfolgend und zugleich abschließend betrachtet werden.

\subsubsection{Ansatzpunkte zur Förderung sinnorientierten Führungshandelns}

Eingedenk der grundsätzlichen Freiheit der geistigen Person mit ihrer Werte- und Sinnbezogenheit handelt es sich bei den im Folgenden aufgezeigten Aspekten nicht um zwingende Voraussetzungen, sondern um förderliche Rahmenbedingungen der Sinnorientierung im Führungskontext. Ohne den Anspruch einer erschöpfenden Aufzählung wollen wir vier Ansatzpunkte kursorisch herausstellen (ähnlich Jung et al. 2018, S. 219f.; unter den „Prädiktoren“ von Sinn in der Arbeit bei Schnell scheint uns die ,sozio-moralische Atmosphäre" hier besonders relevant, Schnell 2016, S. 159f.).

\section{- Sinnbarrieren abbauen und vermeiden}

Wertsichtigkeit und das Erkennen von Sinnmöglichkeiten setzen ein weitgehend angstfreies Agieren mit einem Frei-sein für die Anfragen der Situation (Aufgaben, andere Personen) voraus. Führen mit Druck, Zwang und Angst verengt das Sicht- und Handlungsfeld der beteiligten Personen mit der Konsequenz einer Konzentration auf die eigene Person und das Vermeiden von unangenehmen Situation und Nachteilen. Die „Empfangsbereitschaft" für die Sinnanfragen einer Arbeitssituation kann dann gestört sein.

\section{- Transparenz herstellen}

In hochkomplex-arbeitsteiligen Systemen kann für das Individuum die Beziehung zwischen dem eigenen Handeln und der daraus letztlich entstehenden „Sache“ verlorengehen. Sinnorientierte Führungskommunikation ist aufgerufen, Zusammenhänge über den unmittelbaren Arbeitskontext hinaus darzulegen, damit Einzelne oder eine Gruppe die Einbindung der eigenen Aufgaben und Tätigkeiten in einen größeren (Sinn-)Zusammenhang erkennen können. Aus sinn-theoretischer Sicht hat der 
Sachzielbezug in der Führungskommunikation eine höhere Bedeutung als der, häufig im Vordergrund stehende, Formalzielbezug (z.B. Leistungskennziffern, Umsatz, Rendite).

\section{- Erlebensräume für Freiheit und Verantwortung schaf-} fen

Vielfalt und Ganzheitlichkeit einer Aufgabe und eine möglichst große Selbständigkeit bei der Aufgabenerfüllung erhöhen die Wahrscheinlichkeit, sich angefragt zu fühlen, die eigenen Fähigkeiten bewusst für eine schöpferische Werterealisierung einzusetzen. Mit der wahrgenommenen Freiheit für eigenständiges Handeln eng verknüpft ist das Empfinden von Verantwortung dafür, etwas so und nicht anders gemacht zu haben. Neben diesem Sachbezug wird Verantwortung im Personenbezug durch das Erleben von Gemeinschaft gefördert. Je mehr das Individuum eingebunden ist in die gemeinsame Aufgabenerfüllung mit Anderen, umso größer ist die Wahrscheinlichkeit, von Werten wie Harmonie, gegenseitige Unterstützung, Beteiligungsgerechtigkeit u.a.m. angesprochen $\mathrm{zu}$ werden. Teamartige Arbeitsstrukturen erschweren das Verharren in der Selbstbezogenheit.

\section{- Wertvorstellungen thematisieren}

Über individuelle Wertvorstellungen zu sprechen, möglicherweise auch Übereinstimmungen und Unterschiede zu den offiziell verlautbarten Wertvorstellungen einer Organisation $\mathrm{zu}$ thematisieren, kann eine sinnorientierte Arbeitskultur befördern, ja gegebenenfalls erst die Möglichkeiten zur Werterealisierung am Arbeitsplatz bewusst machen. In einer eher auf „Sachlichkeit“ getrimmten Führungskommunikation ist für die Erörterung von Werten selten Platz. Werte in das Bewusstsein zu bringen, kann die Wertsichtigkeit in der je konkreten Handlungssituation schärfen.

Insbesondere die Einbindung von Wertvorstellungen geht ohne Zweifel in den meisten Arbeitsbeziehungen über das gewohnte Spektrum der Kommunikationsinhalte hinaus und stellt - auch wegen der Subjektivität von Wertvorstellungen - eine Herausforderung dar.

\section{Fazit}

Das anthropologisch-philosophisch begründete, existenzanalytische Verständnis von Sinn bindet Sinnwahrnehmung an den subjektiven und situativen Vorgang von Wertfühlen. In dieser Verbindung ist Sinn ein Motivationsfaktor sui generis. Ihn in die Führungsbeziehung zu integrieren heißt, der Wertebezüglich im Führungshandeln eine deutlich wahrnehmbare Bedeutung einzuräumen. Dafür gibt es, wie mit Bezug auf verschiedene Wertekategorien aufgezeigt werden konnte, eine Vielfalt an Möglichkeiten. Rahmen- gebend gefördert werden kann die je konkrete situative Sinnorientierung durch eher generelle Ansatzpunkte für eine werte- und sinnorientierte Arbeits- und Führungskultur.

Mit der sinnorientierten Aufladung - existenzanalytisch gesprochen: auf geistige Existenz hin ausgerichtet - wird Führungsarbeit zweifelsohne herausfordernder; sie wird nicht per se obsolet. Die Idee einer ,dienenden Führung“ (siehe z.B. Fischer et al. 2019) erhält einen spezifischen Anspruch: Führung im Dienst der Sinnfindung bei den Mitarbeitenden durch Sinnvermittlung und Ermöglichen von Sinnentdeckung. Vorgeschrieben werden kann Sinn nicht. Angesichts der motivationalen Kraft von Sinn (Be-Geisterung) darf vermutet werden, dass sinnorientierte Führung auch einen nennenswerten Beitrag zur Führungsentlastung und zur Stärkung von „Selbstführung“ leisten kann.

Funding Open Access funding provided by Projekt DEAL.

Open Access Dieser Artikel wird unter der Creative Commons Namensnennung 4.0 International Lizenz veröffentlicht, welche die Nutzung, Vervielfältigung, Bearbeitung, Verbreitung und Wiedergabe in jeglichem Medium und Format erlaubt, sofern Sie den/die ursprünglichen Autor(en) und die Quelle ordnungsgemäß nennen, einen Link zur Creative Commons Lizenz beifügen und angeben, ob Änderungen vorgenommen wurden.

Die in diesem Artikel enthaltenen Bilder und sonstiges Drittmaterial unterliegen ebenfalls der genannten Creative Commons Lizenz, sofern sich aus der Abbildungslegende nichts anderes ergibt. Sofern das betreffende Material nicht unter der genannten Creative Commons Lizenz steht und die betreffende Handlung nicht nach gesetzlichen Vorschriften erlaubt ist, ist für die oben aufgeführten Weiterverwendungen des Materials die Einwilligung des jeweiligen Rechteinhabers einzuholen.

Weitere Details zur Lizenz entnehmen Sie bitte der Lizenzinformation auf http://creativecommons.org/licenses/by/4.0/deed.de.

\section{Literatur}

Badura, B. (2018). Über sinnstiftende Arbeit. In B. Badura, A. Ducki, H. Schröder, J. Klose \& M. Meyer (Hrsg.), Fehlzeiten-Report 2018, Sinn erleben - Arbeit und Gesundheit (S. 1-7). Berlin Heidelberg: Springer.

Berschneider, W. (2003). Sinnzentrierte Unternehmensführung. Was Viktor E. Frankl den Führungskräften der Wirtschaft zu sagen hat. Lindau am Bodensee: Orthaus.

Brohm, M. (2017). Werte, Sinn und Tugenden als Steuerungsgrößen in Organisationen. Wiesbaden: Springer.

Busse, E. (2019). Sinnstiftendes Management. Welche Faktoren das Sinnerleben der Mitarbeiter beeinflussen. Zeitschrift Führung + Organisation, 88(2), 130-138.

Busse, R., Kwon, S., Kloep, H.-A., Ghosh, K., \& Warner, M. (2018). Toward a „meaningful self" at the workplace: multinational evidence from Asia, Europe, and North America. Journal of Leadership \& Organizational Studies, 25(1), 63-75.

Felfe, J., Krick, A., \& Reiner, A. (2018). Wie kann Führung Sinn stiften? - Bedeutung der Vermittlung von Sinn für die Gesundheit. In: B. Badura, A. Ducki, H. Schröder, J. Klose \& M. Meyer (Hrsg.), Fehlzeiten-Report 2018, Sinn erleben - Arbeit und Gesundheit (S. 213-223). Berlin - Heidelberg: Springer.

Fink, F., \& Moeller, M. (2018). Purpose Driven Organizations. SinnSelbstorganisation - Agilität. Stuttgart: Schäffer-Poeschel. 
Fintz, A. S. (2014). Leading by Meaning. Die Generation Maybe Sinnorientiert führen. Berlin, Heidelberg: Springer Gabler.

Fischer, H.R., Stahl, H.K., Schettgern, P., \& Schlipat, H. (Hrsg.). (2019). Dienende Führung. Zu einer neuen Balance zwischen ICH und WIR. Berlin: Erich Schmidt.

Förster, A., \& Kreuz, P. (2009). Spuren statt Staub. Wie Wirtschaft Sinn macht (3. Aufl.). Berlin: Econ.

Frankl, V.E. (2006). Der Mensch vor der Frage nach dem Sinn. Eine Auswahl aus dem Gesamtwerk (19. Aufl.). München, Zürich: Piper. Erstveröffentl. 1979

Frankl, V.E. (2007). Ärztliche Seelsorge. Grundlagen der Logotherapie und Existenzanalyse. München: Piper. Erstveröffentl. 1946

Frankl, V.E. (2010). Logotherapie und Existenzanalyse. Texte aus sechs Jahrzehnten (3. Aufl.). Weinheim, Basel: Beltz.

Geramanis, O. (2016). Wider die Verwerkzeugung der Führung - Jenseits der Rationalität. In O. Geramanis \& K. Hermann (Hrsg.), Führen in ungewissen Zeiten - Impulse, Konzepte und Praxisbeispiele (S. 33-48). Wiesbaden: Springer Gabler.

Graf, H. (2007). Die kollektiven Neurosen im Management. Viktor E. Frankl - Wege aus der Sinnkrise in der Chefetage. Wien: Linde.

Grimm, J., \& Grimm, W. (1999). Das deutsche Wörterbuch. OnlineVersion der Universität Trier auf Basis der Buchausgaben von $1984 / 1999$

Hartmann, N. (1949in). Einführung in die Philosophie (5. Aufl.). Hannover: Luise Hanckel. Überarbeitete, vom Verfasser genehmigte Nachschrift der Vorlesung im Sommersemester 1949 in Göttingen

Hartmann, N. (1962). Das Problem des geistigen Seins. Untersuchungen zur Grundlegung der Geschichtsphilosophie und der Geistswissenschaften. Bd. 3. Berlin: De Gruyter.

Jung, R. H. (2019). Besinnt euch! Ein Plädoyer für das Menschliche. Stuttgart: Radius.

Jung, R.H. (2020). Selbstorganisation und die Sinnfrage. In O. Geramanis \& S. Hutmacher (Hrsg.), Der Mensch in der Selbstorganisation. Kooperationskonzepte für eine dynamische Arbeitswelt (S. 41-53). Wiesbaden: Springer Gabler.

Jung, R.H., Heinzen, M., \& Quarg, S. (2018). Allgemeine Managementlehre. Lehrbuch für die angewandte Unternehmens- und Personalführung. Bd. 7. Berlin: Erich Schmidt.

Kuhl, J. (2010). Lehrbuch der Persönlichkeitspsychologie. Motivation, Emotion und Selbststeuerung. Göttingen: Hogrefe.

Laloux, F. (2015). Reinventing Organizations. Ein Leitfaden zur Gestaltung sinnstiftender Formen der Zusammenarbeit. München: Vahlen.

Längle, A. (2008). Existenzanalyse. In A. Längle \& A. Holzhey-Kunz (Hrsg.), Existenzanalyse und Daseinsanalyse (S. 21-179). Wien: facultas.wuv.

Malik, F. (1997). Motivation durch Sinn. Malik on Management, 5(3), 37-48.
Maslow, A.H. (1954). Motivation and personality. New York: Harper $\&$ Brothers.

Maslow, A.H. (1966). Comments on Dr. Frankl's Paper. Journal of Humanistic Psychology, 6(2), 106-112.

Merleau-Ponty, M. (1966). Phänomenologie der Wahrnehmung. Berlin: De Gruyter. Aus dem Französischen übersetzt und eingeführt durch eine Vorrede von Rudolf Boehm

Pattakos, A., \& Dundon, E. (2017). Discovering meaning through the lens of work. Journal of Constructivist Psychology, 30(1), 42-49.

Pircher-Friedrich, A. M. (2019). Mit Sinn zum nachhaltigen Erfolg. Anleitung zur werte- und wertorientierten Führung. Bd. 4. Berlin: Erich Schmidt.

Scheler, M. (1927). Der Formalismus in der Ethik und die materiale Wertethik. Bd. 3. Halle a. d. S: Max Niemeyer.

Scheler, M. (2010). Die Stellung des Menschen im Kosmos (18. Aufl.). Bonn: Bouvier. Erstveröffentlichung 1928

Schnell, T. (2016). Psychologie des Lebenssinns. Berlin, Heidelberg: Springer.

Schnell, T., Höge, T., \& Pollet, E. (2013). Predicting meaning in work: theory, data, implications. The Journal of Positive Psychology, $8(6), 543-554$.

Seidel, E. (1978). Betriebliche Führungsformen. Geschichte, Konzept, Hypothesen, Forschung. Stuttgart: C. E. Poeschel.

Tschirky, H. (1991). Sinn - eine neue Dimension der Führung. io Management-Zeitschrift, 60(1), 27-30.

Zaiser, R. (2004). Management by Meaning: Personalführung und Motivation unter dem Aspekt der Logotherapie - ein Beitrag zum Sinnmanagement. Berlin: LIT.

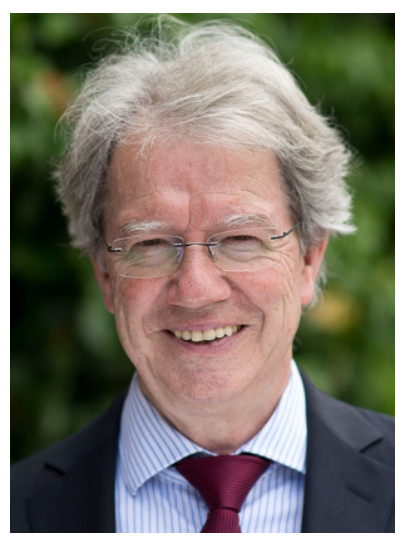

Prof. Dr. Rüdiger Heinrich Jung Prof. Dr. rer. pol.; Studium der Betriebswirtschaftslehre und Sozialpsychologie an der Universität Köln (Diplom 1976); Wiss. Tätigkeit und Promotion mit einer Arbeit über „Selbstorganisation“ an der Universität Siegen (1985); Professur für Management/Führung und Organisationsentwicklung an der Hochschule Koblenz, RheinAhrCampus Remagen (em.); psychotherapeutische Ausbildung; div. mehrjährige Industrietätigkeiten; langjähriger Coach von Führungskräften (www.rhj-institut.de, rhjung@ @rhjinstitut.de). 